\title{
Evidências de Validade com base na Estrutura Interna no Teste dos Contos de Fadas
}

\author{
Blanca Susana Guevara Werlang - Pontificia Universidade Católica do Rio Grande do Sul, Porto Alegre, Brasil \\ Carlos Henrique Sancineto da Silva Nunes - Universidade Federal de Santa Catarina, Florianópolis, Brasil \\ Vivian Roxo Borges - Pontifícia Universidade Católica do Rio Grande do Sul, Porto Alegre, Brasil
}

\begin{abstract}
Resumo
O Teste dos Contos de Fadas/TCF, originalmente grego, avalia aspectos dinâmicos da personalidade de crianças com idades entre seis e onze anos. O presente trabalho teve como objetivo identificar evidências de validade com base na estrutura interna do TCF em uma amostra brasileira. O estudo envolveu 315 crianças da população geral e 167 crianças de grupos clínicos. Os instrumentos utilizados foram: ficha de dados sociodemográficos; Teste Matrizes Progressivas Coloridas de Raven - Escala Especial e o TCF. As crianças da amostra foram localizadas em escolas públicas e privadas e em instituições de saúde e de proteção infantil. Os resultados da análise fatorial exploratória revelaram uma solução com sete fatores que foram avaliados e interpretados de acordo com a proposta teórica do teste. Esses resultados foram satisfatórios e servem como evidências de validade do TCF sob a perspectiva de sua estrutura interna.

Palavras-chave: Avaliação psicológica; Técnica projetiva; Psicologia infantil; Medidas da personalidade; Validade estatística.
\end{abstract}

Validity evidences regarding the internal structure of the Fairy Tales Test

\begin{abstract}
The Fairy Tales Test (FTT), originally from Greece, evaluates dynamic personality aspects of children aged 6 to 11. The current work goal was to identify validity evidences regarding the internal structure of the FTT in a Brazilian sample. The study sample consisted of 315 children from the general population and 167 children from clinical groups. The research made use of a sociodemographic data form, as well as the Raven's Colored Progressive Matrices (CPM), and the FT'T. The children from the sample were recruited from public and private schools and from health and child protection institutions. Regarding the exploratory factorial analysis, it was found a seven-factor solution which were evaluated and interpreted following the test theory proposition. These results are considered satisfactory and indicate internal structure validity for the FTT from a psychometric standpoint. Keywords: Psychological assessment; Projective technique; Child psychology; Measures of personality; Validity statistics.
\end{abstract}

Evidencias de validez basada en la estructura interna del Test de los Cuentos de Hadas

\section{Resumen}

El test de los cuentos de Hadas /TCF, de origen griego, evalúa aspectos dinámicos de la personalidad de niños con edad entre 6 y 11 años. El objetivo de esta investigación fue identificar evidencias de validez basadas en la estructura interna del TCF en una muestra brasileña. Participaron 315 niños de la población general y 167 niños de grupos clínicos. Los instrumentos utilizados fueron: Cuestionario de Datos Sociodemográficos, Test de Matrices Progresivas Coloridas de Raven - Escala Especial y el TCF. Los niños de la muestra fueron localizados en escuelas públicas y particulares y en instituciones de salud y de protección infantil. Los resultados del análisis factorial exploratorio revelaron una solución con siete factores que fueron evaluados e interpretados según la propuesta teórica del TCF. Estos resultados fueron satisfactorios e indican evidencias de validez del TCF, desde la perspectiva de su estructura interna.

Palabras-clave: Evaluación psicológica; Técnica proyectiva; Psicología infantil; Medidas de personalidad; Validez estadística.

\section{Introdução}

Uma parcela expressiva de psicólogos utiliza testes como sua principal ferramenta de trabalho para aferir de forma válida informações essenciais sobre determinado sujeito, tais como padrões de funcionamento, traços de personalidade, aptidões e habilidades. Os testes psicológicos constituem-se, então, como componentes-chave em processos de avaliação psicológica, podendo ser definidos como procedimentos sistemáticos de medida, baseados em processos padronizados, que objetivam acessar características psicológicas dos indivíduos, em amostras de comportamento que sejam relevantes para o seu funcionamento cognitivo e/ou afetivo (Pasquali, 2003; Urbina, 2007). A partir disso, fica evidente que os testes são ferramentas, veículos de acesso a algo, meios para se alcançar um fim.

Existem dois grandes grupos de instrumentos psicológicos, classificados de acordo com sua objetividade e padronização (Anzieu, 1981; Pasquali, 2001): os testes psicométricos (baseados em critérios mais objetivos para quantificar um determinado construto, com respostas que indicam o nível de adaptação do sujeito a padrões estabelecidos) e os testes projetivos (baseados em critérios 
dinâmicos, globais e não observáveis para analisar e/ ou caracterizar um determinado construto). Especificamente sobre os testes projetivos (para crianças ou para adultos), cabe destacar que o principal objetivo desses instrumentos é acessar e compreender os aspectos mais essenciais e dinâmicos da personalidade. Assim, a premissa básica que sustenta esse tipo de teste é a de que toda produção de um sujeito está, de alguma forma e em alguma medida, vinculada às suas capacidades e vivências, que são projetadas ao exterior (Anderson \& Anderson, 1966; Fensterseifer \& Werlang, 2008).

Dois pontos importantes quando se abordam as técnicas projetivas em avaliação psicológica tratam dos contextos em que elas se aplicam e do rol de possibilidades que geram para a avaliação de um sujeito. É certo que sujeitos diferentes, em contextos diferentes, exigirão técnicas adequadas e adaptadas a suas particularidades (Werlang, Villemor-Amaral, \& Nascimento, 2010). Nesse cenário é que se inserem os testes projetivos para crianças.

Como possibilidades de testes projetivos aplicados ao contexto infantil, têm-se os instrumentos gráficos (de fazer desenhos) e os verbais (aperceptivos, associativos, de contar histórias e de completamento de histórias ou palavras). Os testes verbais podem incluir diversos tipos de estímulos. Nesse sentido, encontram-se os testes em que o estímulo são: a) histórias incompletas que exigem que a criança elabore um final para elas (Fábulas de Düss, Teste das Fábulas, Contos de Madeleine Thomas); b) imagens pictóricas que retratam situações do cotidiano ou personagens da literatura infantil em que a criança deve organizar uma história com começo, meio e fim ou deve responder a certas perguntas (Testes Aperceptivos Temáticos - CAT-A, CAT-H, Teste de Montagem de Cenas - MAPS, Teste de Contos de Fadas) e c) imagens abstratas, como as manchas de tintas, em que a criança precisa usar sua capacidade associativa para responder com o que o estímulo se parece (Teste de Rorschach) (Formiga \& Mello, 2000; Werlang \& Cunha, 1993).

O Teste dos Contos de Fadas/TCF compõe o rol de instrumentos projetivos, do tipo aperceptivo temático associativo. O TCF estimula processos inconscientes, criando espaços para a expressão e exploração dos sentimentos e das atitudes de crianças, com base na teoria psicanalítica (Coulacoglou \& Souyouldjoglou, 2005). O arcabouço de instrumentos que se tem hoje no mercado brasileiro é bastante variado, encontrandose uma distribuição entre os testes psicométricos e os testes projetivos, mas quando se pensa em avaliação psicológica de crianças são poucos os instrumentos existentes hoje na lista do Sistema de Avaliação dos Testes Psicológicos/SATEPSI com parecer favorável (Conselho Federal de Psicologia, 2013). Em relação aos testes projetivos infantis, este cenário é ainda mais preocupante.

Para poder operacionalizar um processo de avaliação psicológica é necessário identificar e interpretar informações psicológicas, resultantes de um conjunto de procedimentos confiáveis que possibilitem a realização, por parte do psicólogo, de julgamentos adequados sobre o funcionamento psíquico de determinado sujeito. Neste sentido, para que um instrumento psicológico possa ser utilizado com segurança,a é preciso garantir sua legitimidade e cientificidade. Os testes psicológicos devem apresentar características de precisão e evidências de validade que justifiquem o fato de se ter confiança nos dados que produzem, sendo necessária a adaptação dos mesmos para a realidade na qual serão administrados e avaliados (DeMers e cols., 2000; Pasquali, 2003; Urbina, 2007).

Especificamente sobre o conceito de validade em psicometria, cabe destacar, que este é considerado, no domínio dos testes psicológicos, uma das propriedades métricas mais importantes. A validade é a questão mais fundamental para o desenvolvimento e avaliação dos escores dos testes e de seus respectivos usos. Ela depende diretamente do construto que está sendo medido, dos aspectos que o representam e das evidências que se podem reunir para corroborar qualquer inferência feita sobre uma pessoa, a partir do resultado de testes (American Educational Research Association, American Psychological Association \& National Council on Measurement in Education, 1999; Primi, Muniz \& Villemor-Amaral, 2009; Urbina, 2007). A garantia de cientificidade dos instrumentos psicológicos deve ancorar-se em pesquisas empíricas sobre a validade, com a aplicação de métodos científicos adequados (Boorsboom, Mellenbergh \& van Heerden, 2004; Primi, Muniz \& Nunes, 2009).

Se um instrumento não possui validade não há segurança, então, de que as interpretações sobre as características psicológicas dos indivíduos, sugeridas por suas respostas na testagem, sejam legítimas. Nessa situação, não se tem certeza sobre o que o instrumento psicológico efetivamente avalia e quão bem ele faz isso e, portanto, o seu uso na prática profissional pode ficar impedido tanto ética quanto tecnicamente (Primi, Muniz \& Nunes, 2009).

A validade é um conceito único, não existindo diferentes tipos de validade, mas sim diferentes aspectos 
(fontes de evidência), que podem ser utilizados na avaliação de possíveis interpretações de escores/variáveis (Urbina, 2007). A validade é definida como se referindo "ao grau em que evidência e teoria sustentam as interpretações dos escores dos testes, vinculados aos usos propostos dos testes" (American Educational Research Association, American Psychological Association \& National Council on Measurement in Education, 1999, p. 9).

Uma fonte de evidência de validade é aquela com base na estrutura interna do teste, baseada nas correlações entre itens que avaliam o mesmo construto e entre subtestes que avaliam construtos similares. Essa evidência de validade enfoca as variáveis internas ao próprio teste, trabalhando na perspectiva da covariância entre partes do teste.

Considerando todos os apontamentos mencionados e entendendo que é de fundamental importância poder dispor de instrumentos adequados e confiáveis em relação ao que se quer avaliar, e que o TCF pode ser um valioso auxílio para o psicólogo clínico que trabalha com crianças em processos de avaliação psicológica, julga-se pertinente verificar suas qualidades psicométricas. Para isso, este artigo apresenta um estudo de evidência de validade com base na estrutura interna das variáveis do Sistema de Categorização de Respostas do TCF utilizando, para tanto, a análise fatorial. Para que esse objetivo pudesse ser alcançado, desenvolveu-se uma pesquisa quantitativa, de tipo transversal, incluindo-a na categoria de estudos instrumentais (de propriedades psicométricas), como expressam Montero e León (2005).

\section{Método}

\section{Amostra}

A amostra foi constituída por 482 crianças com idades entre 6 e 11 anos e incluiu um grupo de crianças da população geral (não clínico) e um grupo clínico. O grupo amostral da população geral contou com 315 crianças, meninos e meninas, que frequentavam instituições escolares públicas e privadas da cidade de Porto Alegre. O grupo clínico foi composto por crianças com diagnóstico médico-clínico, clínico-psiquiátrico e por crianças vítimas de abuso sexual intrafamiliar (total de 167 crianças). Esse grupo foi localizado a partir de uma população de crianças com diagnóstico já formulado ou situação de vida já definida, admitidas em instituições hospitalares, clínicas-escola e instituições de proteção infantil. Mas especificamente o grupo amostral clínico contou com 30 crianças com diagnóstico de diabetes mellitus, 28 com transtorno depressivo, 23 com transtorno de conduta, $31 \mathrm{com}$ transtorno de déficit de atenção/hiperatividade/TDAH, 23 crianças com transtornos da aprendizagem e 32 crianças vítimas de abuso sexual intrafamiliar.

Para a inclusão dos sujeitos no grupo clínico, os critérios foram: concordância dos pais e/ou responsáveis com a participação do(a) filho(a) no estudo, ausência de comprometimento intelectual e apresentação de um dos diagnósticos ou situação de interesse, conforme descrito acima. Para a inclusão dos sujeitos no grupo da população geral, os dois primeiros critérios foram os mesmos, acompanhados de ausência de diagnóstico psicopatológico, médico e/ou de vivência de abuso sexual intrafamiliar.

\section{Instrumentos}

A fim de obter dados que caracterizassem os participantes do estudo, foi utilizada uma ficha de dados sociodemográficos, que continha informações sobre sexo, idade, história escolar e de saúde da criança, seu núcleo familiar e sua condição socioeconômica. Para excluir casos com suspeita de comprometimento intelectual (abaixo do percentil 25) foi administrado, de forma individual, o Teste Matrizes Progressivas Coloridas de Raven - Escala Especial (Angelini, Alves, Custódio, Duarte \& Duarte, 1999). A administração foi realizada seguindo as instruções usuais que constam no manual, prevendo-se uma duração média de 15 a 30 minutos. O Teste de Matrizes Progressivas foi desenvolvido pelo psicólogo J. C. Raven e foi criado como medida do fator "g", com base no referencial de Spearman (1927). Como uma tarefa a ser cumprida, pode ser descrito como um teste de completamento e, em termos do tipo de item, é um teste de escolha entre soluções alternativas. $\mathrm{O}$ caderno a ser administrado é dividido em séries de matrizes ou desenhos que apresentam um problema introdutório, cuja solução é clara, fornecendo um padrão para a tarefa, que se torna progressivamente mais difícil. A Escala Especial compreende três séries (A, Ab, e B). As respostas são classificadas como positivas ou negativas e cada resposta certa recebe um ponto; o total de pontos é o escore obtido pelo sujeito. Esse escore é transformado em percentil através do uso de uma tabela específica em associação com a idade do sujeito.

$\mathrm{O}$ instrumento-alvo deste estudo foi o Teste dos Contos de Fadas/TCF, organizado por Coulacoglou (1995). O TCF é composto por 21 cartões com 
personagens e imagens de cenas vinculadas a, basicamente, três contos de fadas (Chapeuzinho Vermelho, Branca de Neve e João e o Pé de Feijão), agrupados em sete séries, compostas por três cartões cada. Os cartões apresentam desenhos de personagens dos contos de fadas referidos acima e cenas de duas dessas histórias. Em relação à sua aplicação, o TCF é administrado individualmente, em um único encontro, de aproximadamente 45 minutos. Antes da administração, o examinador deve verificar a familiaridade da criança com os contos que estão presentes no teste. Portanto, é essencial que a criança conheça, a história da Chapeuzinho Vermelho, da Branca de Neve e alguma história de gigantes, como, por exemplo, João e o Pé de Feijão ou O Pequeno Polegar. Se a criança não estiver familiarizada com os contos mencionados, aconselha-se remarcar a administração do teste para dali a uma semana, após a explanação do conto.

Coulacoglou (2008) propõe a análise de 30 variáveis (ver Tabela 1), que podem ser identificadas nas respostas dadas ao teste. Essas variáveis estão alocadas em cinco grupos maiores, denominados pela autora de componentes da personalidade: desejos e necessidades (desejo por coisas materiais, desejo de superioridade, desejo de ajudar, necessidades orais, necessidade de afiliação, necessidade de aprovação, necessidade de afeto, necessidade de proteção); impulsos (preocupação sexual, bizarro, agressão oral, agressão impulsiva, agressão como dominância, agressão instrumental, agressão como defesa, agressão como retaliação, agressão por inveja e agressão por ciúme), relações objetais (relação com a mãe e relação com o pai), estados emocionais (medo de agressão, ansiedade, depressão) e funções do ego (ambivalência, autoestima, moralidade, senso de propriedade, senso de privacidade, adaptação ao conteúdo do conto e repetição). Coulacoglou $(1995,2008)$ afirma que os contos de fadas fazem parte do imaginário das crianças enfocando temas que contêm situações universais e refletem valores tradicionais como: amor, amizade, inveja, ajuda, violência, morte e ressurreição. Assim sendo, o TCF estimula os processos inconscientes explorando sentimentos e atitudes e avalia 30 dimensões da personalidade infantil que são consideradas variáveis a serem pontuadas a partir das respostas da criança ao teste. Na Tabela 1 podem ser visualizadas as 30 variáveis, assim como as subdivisões das variáveis: ambivalência e ansiedade. $\mathrm{Na}$ Tabela 2 constam os mecanismos de defesas.

As variáveis que compõem o Sistema de Categorização de Respostas do TCF devem ser pontuadas com base na sua intensidade (Coulacoglou, 1995, 2002a, 2002b, 2008). A maioria delas é classificada numa escala de pontuação que varia entre 1 e 3 , em que 1 significa baixa intensidade e 3, alta. Já no caso de certas variáveis, tais como autoestima, relacionamento com a mãe e relacionamento com o pai, a pontuação é positiva $(+1)$ ou negativa $(-1)$, dependendo da natureza da resposta. Ainda, as respostas bizarras e repetições são pontuadas como presentes ou ausentes (0 ou 1$)$, e em relação à variável adaptação ao conteúdo do conto, a pontuação atribuída varia de 0 a 3. Para esta última variável deve ser atribuída uma pontuação 0 quando nenhuma ação, qualquer que seja, estiver envolvida ou implicada na resposta, ou quando a criança responder: "Eu não sei" ou "Eu não lembro", ou dá respostas "neutras", sem significado.

Além da proposta interpretativa quantitativa, baseada na comparação do número de escores baixos versus os altos, o TCF oferece subsídios para realizar uma interpretação qualitativa que inclui: observações comportamentais da criança durante a aplicação do teste, comentários sobre as figuras, rejeição aos cartões, mudança na ordem dos cartões, nível de concentração da criança (dificuldade em ficar sentada, remexer-se, demonstrar impaciência) e sua habilidade verbal (gramática, sintaxe, vocabulário, palavras ou expressões idiossincráticas).

Especificamente sobre os mecanismos de defesa, observa-se que os mais frequentes nas respostas das crianças ao TCF são: anulação, negação, projeção, formação reativa, repressão, cisão, racionalização, identificação projetiva, regressão, deslocamento, compensação, agressão contra a própria pessoa, identificação com o agressor, introjeção, atuação e dissociação (Coulacoglou, 1995, 2002a, 2002b, 2008).

\section{Procedimentos para a coleta de dados}

Para composição da amostra da população geral, foram contatadas instituições escolares públicas e privadas de Porto Alegre, para obter a autorização necessária para a testagem das crianças. Previamente à administração dos instrumentos, foi encaminhada uma carta aos pais e/ou responsáveis pelos alunos com o objetivo de explicar a natureza e relevância do trabalho a ser desenvolvido, bem como obter deles a autorização para a participação da criança. Além dessa carta, também foi encaminhada a ficha de dados sociodemográficos e o Termo de Consentimento Livre e Esclarecido para os pais e/ou responsáveis pela criança. De posse do consentimento de participação e da ficha de dados, a 
Tabela 1. Configuração das variáveis do sistema de categorização de respostas do Teste dos Contos de Fadas/TCF, conforme os cinco componentes da personalidade

\begin{tabular}{|c|c|c|}
\hline Componentes da Personalidade & Variáveis & Intensidade das Variáveis \\
\hline \multirow[t]{8}{*}{ Desejos e necessidades } & 1) Desejo por coisas materiais (DCM) & $1,2,3$ \\
\hline & 2) Desejo de superioridade (DSup) & $1,2,3$ \\
\hline & 3) Desejo de ajudar (DA) & $1,2,3$ \\
\hline & 4) Necessidades orais (NO) & $1,2,3$ \\
\hline & 5) Necessidade de afiliação (NAfil) & $1,2,3$ \\
\hline & 6) Necessidade de aprovação (NApr) & $1,2,3$ \\
\hline & 7) Necessidade de afeto (NAft) & $1,2,3$ \\
\hline & 8) Necessidade de proteção (NPro) & $1,2,3$ \\
\hline \multirow[t]{10}{*}{ Impulsos } & 9) Preocupação sexual (PSex) & $1,2,3$ \\
\hline & 10) Respostas bizarras (B) & Presença ou ausência \\
\hline & 11) Agressão oral (AO) & $1,2,3$ \\
\hline & 12) Agressão impulsiva (AgrImp) & $1,2,3$ \\
\hline & 13) Agressão como dominância (AgrDom) & $1,2,3$ \\
\hline & 14) Agressão instrumental (AgrIns) & $1,2,3$ \\
\hline & 15) Agressão como defesa (AgrDef) & $1,2,3$ \\
\hline & 16) Agressão por inveja (AgrInv) & $1,2,3$ \\
\hline & 17) Agressão por ciúme (AgrCiu) & $1,2,3$ \\
\hline & 18) Agressão como retaliação (AgrRet) & $1,2,3$ \\
\hline \multirow[t]{7}{*}{ Funções do ego } & $\begin{array}{l}\text { 19) Ambivalência (AMB) [indecisão, dúvida, } \\
\text { conflito, alternativa, hesitação, incerteza] }\end{array}$ & $1,2,3$ \\
\hline & 20) Auto estima (AE) & $-1 /+1$ \\
\hline & 21) Moralidade (Mor) & $1,2,3$ \\
\hline & 22) Senso de propriedade (SPro) & $1,2,3$ \\
\hline & 23) Senso de privacidade (SPriv) & $1,2,3$ \\
\hline & 24) Adaptação ao conteúdo do conto (ACCF) & $0,1,2,3$ \\
\hline & 25) Repetição (Rep) & Presença ou ausência \\
\hline \multirow[t]{3}{*}{ Estados emocionais } & 26) Medo de agressão (MA) & $1,2,3$ \\
\hline & $\begin{array}{l}\text { 27) Ansiedade (Ans) [perda, doença, dano, } \\
\text { insegurança, rejeição, castigo, desaprovação, } \\
\text { trabalho duro, autoimagem, morte, solidão, } \\
\text { preocupação com os outros, reprovação, } \\
\text { privação, indiferença, incapacidade, proibição] }\end{array}$ & $1,2,3$ \\
\hline & 28) Depressão (D) & $1,2,3$ \\
\hline \multirow[t]{2}{*}{ Relações objetais } & 29) Relação com a mãe (Rel/Mae) & $-1 /+1$ \\
\hline & 30) Relação com o pai (Rel/Pai) & $-1 /+1$ \\
\hline
\end{tabular}


Tabela 2. Configuração dos mecanismos de defesa incluídos, neste estudo, no Sistema de Categorização de Respostas do Teste dos Contos de Fadas/TCF

\begin{tabular}{l|l}
\hline Mecanismos de Defesa & Intensidade das Variáveis \\
\hline Anulação & \\
Negação & \\
Projeção & \\
Formação Reativa & \\
Repressão & Presença ou ausência \\
Cisão & \\
Racionalização & \\
Identificação Projetiva & \\
Regressão & \\
Deslocamento & \\
Compensação & \\
Agressão contra a própria pessoa & \\
Identificação com o agressor & \\
Introjeção & \\
Acting out & \\
Dissociação & \\
\hline
\end{tabular}

Fonte: Coulacoglou (1995, 2002a, 2002b, 2008, 2009).

administração dos instrumentos foi realizada de forma individual, em um encontro (Teste Raven e TCF), na própria instituição, durante o período escolar.

Para a coleta de dados das crianças do grupo amostral clínico (médico-clínica, clínico-psiquiátrica e de vítimas de abuso sexual intrafamiliar), foram contatadas instituições hospitalares, clínicas-escola e instituições de proteção infantil, que atendessem crianças com os diagnósticos ou situações anteriormente mencionados. Esclarecimentos e reuniões com os coordenadores e/ou chefias desses locais foram particularmente importantes e necessárias para se estabelecer um plano adequado para a operacionalização do estudo. Assim, diante da concordância das instituições, os pais e/ou responsáveis pelos pacientes foram inicialmente comunicados sobre o estudo pelo profissional responsável pelo atendimento e, caso concordassem em participar do mesmo, assinavam o Termo de Autorização, que constava da autorização dos mesmos para o encaminhamento do nome de seu (sua) filho(a) para os pesquisadores. De posse disso, era possível agendar uma entrevista com os pais e/ou responsáveis pela criança, na qual eram explicitadas a natureza e a relevância do trabalho a ser desenvolvido. Nessa entrevista também se assinava o Termo de Consentimento Livre e Esclarecido, e se preenchia a ficha de dados sociodemográficos. Após a entrevista com os pais e/ou responsáveis, a criança foi submetida aos instrumentos (Teste Raven e TCF) em um único encontro, na própria instituição em que o participante estava inserido.

A coleta dos dados foi realizada após aprovação do projeto pelo Comitê de Ética em Pesquisa da Pontifícia Universidade Católica do Rio Grande do Sul/PUCRS e pelo Comitê de Ética em Pesquisa do Hospital Nossa Senhora da Conceição/GHC. A coleta dos dados de toda a amostra foi seguida da digitação das respostas de cada criança ao TCF, da classificação dos instrumentos a da inclusão dos dados em banco específico, para processamento e análise posterior.

\section{Procedimentos para a análise dos dados}

Todas as informações coletadas com os instrumentos aplicados foram organizadas em banco de dados, no programa estatístico Statistical Package for the Social Sciences SPSS, versão 17.0. Inicialmente, para conhecer as características sociodemográficas e cognitivas, foi realizada uma análise descritiva dos dados, através de cálculo de frequências e porcentagens. Após a administração do Teste dos Contos de Fadas, todos os protocolos foram analisados e as verbalizações das crianças, para cada um dos 21 desenhos do teste, foram classificadas com base: 1) nas variáveis e subvariáveis do 
Sistema de Categorização de Respostas e 2) na identificação dos mecanismos de defesas. Para o processo de tabulação foi adotada uma estrutura de banco de dados na qual os índices gerados a partir das respostas de cada participante foram registrados individualmente para cada um dos 21 desenhos.

Para identificar a evidência de validade com base na estrutura interna das variáveis e subvariáveis do Sistema de Categorização de Respostas do TCF foi utilizado o procedimento de análise fatorial exploratória, com a utilização do método de extração por componentes principais com rotação varimax. Foram considerados para a análise os índices obtidos a partir das respostas de todas as crianças da amostra $(\mathrm{N}=482)$ ao teste. A análise fatorial é um método criado para se descobrir agrupamentos de variáveis com base na estrutura interna de intercorrelações que, em certas condições, podem ser indicativos de dimensões psicológicas latentes (Villemor-Amaral \& Primi, 2009). Nesse sentido, ela pode ser aplicada ao TCF com o objetivo de se conhecer quais as variáveis e quais os mecanismos de defesa que, agrupados, podem exemplificar características de personalidade das crianças da amostra $(\mathrm{N}=482)$. $\mathrm{O}$ aspecto mais importante, nesse sentido, é verificar se os fatores extraídos (mesmo apresentando qualidades psicométricas aceitáveis) fazem sentido a partir da modelo teórico do teste. Para fins da realização da análise fatorial e, posteriormente, para estimar os resultados das crianças em cada fator identificado, foi gerada uma base de dados agregada, na qual para todas as crianças os resultados encontrados para cada variável nos 21 desenhos foram somados. Assim, a base agregada apresenta, para as variáveis pontuadas em termos de "ausência e presença", a quantidade de vezes em que tal indicador foi identificado nas respostas das crianças para todas as cartas. Para as variáveis pontuadas em termos de intensidade ou severidade, a base agregada apresentou um índice total de tal indicador para as respostas avaliadas. Vale notar que algumas das variáveis são invertidas, ou seja, apresentam correlações negativas com o fator em que estão alocadas. Tais variáveis foram transformadas para a análise fatorial e o cálculo do resultado dos participantes nos fatores identificados.

\section{Resultados}

\section{Características sociodemográficas}

Considerando aspectos que caracterizam a amostra da população geral a partir da ficha de dados sociodemográficos, observou-se que das 315 crianças, $203(64,4 \%)$ frequentavam escolas públicas e 112 $(35,6 \%)$ escolas particulares. A idade média das 315 crianças foi de 8,57 anos (DP=1,64), sendo $143(45,4 \%)$ do gênero masculino e $172(54,6 \%)$ do gênero feminino. Em relação ao ano escolar que essas crianças frequentavam, o $2^{\circ}(64,20,4 \%)$ e o $4^{\circ}(71,22,3 \%)$ anos do ensino fundamental foram os mais prevalentes. Das 315 crianças, a maioria, 298 (94,6\%) não teve repetência escolar, resultado este em consonância com o fato de que, na opinião dos pais e/ou responsáveis, um número expressivo delas apresenta um desempenho escolar entre ótimo (196, 62,6\%) e bom (102, 32,6\%). Ainda, o resultado obtido no Teste Matrizes Progressivas Coloridas de Raven - Escala Especial, aponta que todas as 315 crianças apresentaram capacidade intelectual na média ou acima da média nesse teste.

Sobre o núcleo familiar em que esses participantes estão inseridos, os dados coletados revelam que a maioria $(201,63,8 \%)$ das crianças da população geral reside com o pai, com a mãe e com, pelo menos, um irmão. Em relação à classe socioeconômica desse grupo amostral não clínico, a classe B (226, 71,7\%) foi a mais prevalente. Ainda, a respeito da presença de doença física, das 315 crianças pesquisadas, a maioria não apresenta doença física (302, 95,9\%). Das crianças que apresentam alguma doença física, 13 (4,1\%) sofrem de doenças respiratórias (asma ou bronquite). Não há referência, nesse grupo da população geral, da presença de transtornos psicológicos. Perguntou-se, igualmente, se os participantes fazem ou fizeram tratamento especializado, e os dados coletados revelaram que 49 (15,6\%) tratam a asma ou bronquite, e 25 (7,9\%), sem especificar o motivo, são acompanhados por psicólogos, em sessões de psicoterapia. Quanto ao uso de medicamentos, $20(6,3 \%)$ participantes referem utilizar medicamentos para o tratamento de asma ou bronquite.

No que se refere à amostra clínica, composta por 167 crianças, a idade média identificada foi de 9,02 anos $(\mathrm{DP}=1,55)$ sendo 110 crianças $(65,8 \%)$ do gênero masculino e 57 (34,1\%) do gênero feminino. Em relação ao ano escolar que as crianças da amostra clínica frequentavam, a distribuição mais prevalente entre os diferentes grupos foi a seguinte: diabetes $\left(5^{\circ}\right.$ ano, 7 crianças, $23,3 \%$ ), transtorno depressivo ( $3^{\circ}$ ano, 10 crianças, 35,7\%), transtorno de conduta (4 ano, 7 crianças, 30,4\%), transtorno de déficit de atenção/ hiperatividade ( $3^{\circ}$ ano, 9 crianças, $29,0 \%$ ), transtorno de aprendizagem ( $3^{\circ}$ ano, 7 crianças, $30,4 \%$ ) e abuso sexual $\left(2^{\circ}, 3^{\circ}\right.$ e $4^{\circ}$ anos, 21 crianças, $\left.65,7 \%\right)$. 
Das 167 crianças do grupo clínico, 44 (26,3\%) repetiram alguma série, mais especificamente: 3 $(10,0 \%)$ com diabetes, $6(21,4)$ com transtorno depressivo, $7(30,4)$ com transtorno de conduta, $13(41,9 \%)$ com transtorno de déficit de atenção/hiperatividade, 5 $(21,7 \%)$ com transtorno de aprendizagem e 10 (31,3\%) crianças vítimas de abuso sexual intrafamiliar. Ainda, as crianças com TDAH $(10,35,7 \%)$, juntamente com as crianças com transtorno depressivo $(13,41,9 \%)$ e vítimas de abuso sexual $(16,50,0 \%)$, na opinião dos pais e/ ou responsáveis, apresentam um desempenho escolar regular.

Em relação aos dados obtidos no Teste Matrizes Progressivas Coloridas de Raven - Escala Especial, observa-se que a maioria das crianças $(154,92,2 \%)$ do grupo clínico como um todo apresentam um desempenho intelectual médio ou acima da média. Considerando os diversos subgrupos, foi identificado desempenho intelectual abaixo da média em $1(3,7 \%)$ criança com transtorno depressivo, 1 (3,3\%) com transtorno de déficit de atenção/hiperatividade, $4(17,4 \%)$ com transtorno de aprendizagem e $7(22,6 \%)$ crianças vítimas de abuso sexual.

Sobre o núcleo familiar em que as 167 crianças da amostra clínica estão inseridas, os dados coletados revelam que $65(38,9 \%)$ crianças residem com o pai, com a mãe e com, pelo menos, um irmão. Em relação à classe socioeconômica dos diferentes grupos clínicos a classe $\mathrm{B}$ também foi a mais prevalente. Com relação à presença de doença física, as crianças com transtorno depressivo $(4,14,3 \%)$, transtorno de conduta $(2$, $8,7 \%$ ), transtorno de déficit de atenção/hiperatividade $(4,12,9 \%)$ e vítimas de abuso sexual $(7,21,9 \%)$ sofrem de anemia, alergias ou doenças respiratórias (asma ou bronquite). Todas as crianças com diabetes mellitus (30, $100 \%$ ) fazem tratamento médico, de controle da diabetes com insulinoterapia. As crianças com vivência de abuso sexual encontram-se todas em acompanhamento psicoterápico.

Evidências de validade com base na estrutura interna das variáveis do teste por meio da análise fatorial

Por meio da análise fatorial exploratória (por componentes principais e rotação varimax) da base agregada de todas as crianças da amostra dadas ao teste, verificou-se primeiramente soluções fatoriais com 5,6 , 7, 8, 9 e 10 fatores. Todas essas soluções foram analisadas levando-se em conta a pertinência dos fatores encontrados (quanto ao conteúdo) e as características psicométricas das dimensões identificadas. A solução com 7 fatores mostrou-se a mais pertinente em termos de intercorrelações (de variáveis, subvariáveis e mecanismos de defesa) com características psicométricas adequadas, explicando $25,0 \%$ da variância total (ver Tabela 3).

Ainda, como regra para a inclusão das variáveis nos fatores foi determinado que teriam que apresentar no mínimo 0,30 de carga fatorial, não havendo a ocorrência de variáveis com cargas fatoriais acima da linha de corte em mais de um fator. Cabe mencionar, ainda, que essa análise fatorial foi realizada com toda a amostra (482 crianças), ou seja, incluiu as crianças do grupo não clínico e clínico, diferenciando-se do estudo original grego, que foi desenvolvido somente com crianças da população geral (Coulacoglou, 2009). Na Tabela 3 constam as matrizes das cargas fatoriais das variáveis nas sete dimensões resultantes da análise.

\section{Discussão e Considerações finais}

A análise dos sete fatores (agrupamentos) permite a exploração dos processos mais gerais subjacentes à formação das interpretações das perguntas aos 21 cartões (com personagens e imagens de cenas) do TCF pelas crianças da amostra. Fazendo uma análise exploratória, segue abaixo a explicação de cada um dos fatores identificados:

O fator 1, a partir das variáveis agrupadas, recebeu o título de "ansiedade". Este fator agrupou as variáveis: ansiedade doença (preocupação de ficar doente ou de que alguém próximo fique doente), ansiedade morte (preocupação com a possibilidade de morrer ou de que alguém próximo morra), ansiedade autoimagem (preocupação em relação a atributos pessoais, comportamentais e físicos), relação com a mãe (relacionamento mãe/filho), ansiedade privação (preocupação com a perda ou restrição simbólica ou concreta de bens, vantagens e direitos) e ansiedade castigo (preocupação de ser ou de que alguém próximo seja sancionado ou reprimido por uma conduta considerada incorreta). Este fator associa cinco variáveis vinculadas à emoção ansiedade (presentes no componente da personalidade Estados Emocionais) propostas pelo TCF, expressando uma intercorrelação de pensamentos relacionados a preocupações com perigos eminentes e eventos angustiantes, ligados à qualidade do relacionamento percebido com a mãe.

O fator 2 agrupou variáveis que na sua associação podem retratar o que se denominou como "necessidade de afeto e aspectos depressivos". Este fator agrupou as 
Tabela 3. Matriz de cargas fatoriais da análise das respostas $(\mathrm{N}=482)$.

\begin{tabular}{|c|c|c|c|c|c|c|c|}
\hline & 1 & 2 & 3 & 4 & 5 & 6 & 7 \\
\hline Ansiedade doença &, 524 & & & & & & \\
\hline Ansiedade morte & , 480 & & & & & & \\
\hline Ansiedade autoimagem & , 479 & & & & & & \\
\hline Relação com a mãe & ,407 & & & & & & \\
\hline Ansiedade privação & ,388 & & & & & & \\
\hline Ansiedade castigo & ,364 & & & & & & \\
\hline Ambivalência conflito & & ,464 & & & & & \\
\hline Necessidade aprovação & & ,445 & & & & & \\
\hline Ansiedade rejeição & & ,437 & & & & & \\
\hline Necessidade afeto & & ,436 & & & & & \\
\hline Depressão & & ,412 & & & & & \\
\hline Necessidade afiliação & & ,359 & & & & & \\
\hline Ansiedade solidão & & ,339 & & & & & \\
\hline Ansiedade indiferença & & ,312 & & & & & \\
\hline Ansiedade incapacidade & & ,302 & & & & & \\
\hline Agressão como defesa & & & ,476 & & & & \\
\hline Deslocamento & & & ,423 & & & & \\
\hline Necessidade de proteção & & & ,397 & & & & \\
\hline Projeção & & & ,363 & & & & \\
\hline Ansiedade dano & & &, 317 & & & & \\
\hline Desejo de superioridade & & & &, 522 & & & \\
\hline Negação & & & & , 470 & & & \\
\hline Autoestima & & & & ,459 & & & \\
\hline Formação reativa & & & & ,398 & & & \\
\hline Anulação & & & & ,321 & & & \\
\hline Agressão dominância & & & & ,321 & & & \\
\hline Necessidades orais & & & & & ,466 & & \\
\hline Desejo por coisas materiais & & & & & ,411 & & \\
\hline Ansiedade trabalho duro & & & & &, 341 & & \\
\hline Senso de propriedade & & & & & ,337 & & \\
\hline Agressão inveja & & & & & & ,491 & \\
\hline Agressão retaliação & & & & & & , 480 & \\
\hline Repressão & & & & & & , 426 & \\
\hline Ambivalência dúvida & & & & & & &, 520 \\
\hline Ambivalência indecisão & & & & & & &, 518 \\
\hline Ambivalência alternativa & & & & & & & ,395 \\
\hline
\end{tabular}

Método de extração: Análise de Componente Principal.

Método de rotação: varimax com normalização kaiser

Rotação convergiu em 17 interações. 
variáveis: ambivalência conflito (emoções opostas e conflitantes), necessidade de aprovação (necessidade de encorajamento para as ações para manter e aumentar a autoestima), ansiedade rejeição (preocupação com a perda de amor e afeto), necessidade de afeto (necessidade de oferecer e receber amor e afeto), depressão (sentimentos de tristeza, choro, desânimo, insatisfação e solidão), necessidade de afiliação (chegar perto e cooperar de forma prazerosa ou recíproca com algum aliado para satisfazer ou ganhar afeto), ansiedade solidão (preocupação de ficar sozinho, desamparado, de se separar), ansiedade indiferença (preocupação de não ser reconhecido, aceito, de pertencer) e ansiedade incapacidade (preocupação de não ser capaz). Este fator, com este agrupamento de variáveis relacionadas, parece indicar respostas vinculadas a manifestação de sentimentos e pensamentos negativos (depressivos) e, em função disso, acompanhados de necessidades de amor, afeto e encorajamento.

O fator 3 deste estudo, a partir das variáveis agrupadas, recebeu o título de "necessidade de proteção frente ao dano". Este fator agrupou as variáveis: agressão como defesa (necessidade de se proteger ou proteger alguém diante do perigo imediato), deslocamento (redirecionamento de sentimento ou reação acerca de um objeto para outro, geralmente menos ameaçador), necessidade de proteção (necessidade de buscar segurança e ajuda daqueles percebidos como mais fortes ou poderosos), projeção (impulsos, sentimentos ou pensamentos não tolerados pelo ego são atribuídos a outros), e ansiedade dano (preocupação de sofrer algum dano fisico ou emocional). Este agrupamento parece indicar a associação de respostas que envolvem preocupação frente a danos, perigos, ameaças, internas e externas, com a necessidade de proteção diante disso, mesmo que seja de forma agressiva (como defesa, sobrevivência) ou utilizando mecanismos defensivos como o deslocamento e a projeção.

O fator 4 agrupa variáveis que podem responder à denominação de "insegurança e afirmação". Este fator incluiu as variáveis: desejo de superioridade (autoengrandecimento, desejo de se destacar, de ser único), negação (transforma, distorce, minimiza ou recusa impulsos agressivos e da realidade externa considerados ameaçadores em seu oposto), autoestima (autoaceitação, autorespeito, autovalorização, preocupação com a aparência, sucesso ou fracasso, aprovação ou desaprovação), formação reativa (sentimentos e impulsos inaceitáveis são substituídos por seus extremos opostos), anulação (retificação ou negação de pensamentos, sentimentos ou ações consideradas inaceitáveis, defesa contra desejos narcisistas, baixa autoestima, medo de agressão e agressão) e agressão como dominância (necessidade de dominar ou provar algo a si mesmo, maneira de aprender a controlar o ambiente e adquirir autoconfiança). Este fator sugere que diante de uma autoestima prejudicada a criança pode se defender (pela negação, formação reativa ou anulação) ou agir de forma contrária, mostrando-se superior ou agredindo para dominar.

O fator 5 , a partir das variáveis agrupadas, permite estabelecer a denominação de "necessidades concretas". Este fator agrupou as variáveis: necessidades orais (necessidade real ou simbólica de comer/beber, simbolizam a incorporação de um objeto), desejo por coisas materiais (aquisição de dinheiro, brinquedos, roupas, carro, jóias ou prazeres materiais, como levar uma vida abastada), ansiedade trabalho duro (preocupação em trabalhar bastante ou cansaço devido ao processo de trabalho) e senso de propriedade (necessidade de controlar o seu território e a posse de objetos pessoais de valor emocional ou material). Este fator reflete a necessidade da criança de adquirir coisas materiais e mantê-las consigo, como uma forma de compensar as necessidades internas mais primitivas insatisfeitas (orais).

O fator 6, a partir das variáveis agrupadas, recebeu o título de "agressão e defesa". Este fator agrupou as variáveis: agressão por inveja (necessidade de causar dano a indivíduos que aparentam ser melhores ou ter mais vantagens do que o agressor em algum aspecto; descontentamento pessoal devido à posse que outras pessoas têm de algo que se gostaria de ter), agressão como retaliação (agressão devido à vingança ou punição, após um prejuízo ter sido causado, depreciação do ego e da autoestima) e repressão (esquecimento "proposital" ou falha em reconhecer desejos ou pensamentos proibidos ou perturbadores). Este agrupamento parece caracterizar respostas que sugerem interação entre conteúdos agressivos mobilizados pela inveja e/ou suposto prejuízo na autoestima, sem reconhecer a proibição ou perturbação implícita nesses conteúdos agressivos.

E o último fator, o 7, a partir das variáveis agrupadas, recebeu a denominação de "ambivalência". Este fator agrupou as variáveis: ambivalência dúvida (incerteza ou descrença em opiniões e credos), ambivalência indecisão (dificuldade em tomar uma decisão ou fazer uma escolha) e ambivalência alternativa (mais do que uma alternativa vinculada às escolhas). Este agrupamento 
reúne três tipos da variável ambivalência (do componente de personalidade Funções do Ego) que associam respostas que indicam divisão, conflito e dificuldades para integrar escolhas, opiniões ou aspectos de um mesmo objeto ou pessoa.

Os componentes da análise fatorial identificados mostram agrupamentos de variáveis bastante coerentes quanto a sua associação, permitindo visualizar conexões teoricamente viáveis com o proposto por Coulacoglou (1995, 2002a, 2002b, 2008, 2009) para o TCF, considerando que este instrumento possibilita compreender o funcionamento de alguns aspectos dinâmicos da personalidade de crianças, com base na teoria psicanalítica. Este estudo demonstra que o TCF dispõe de qualidades psicométricas de validade com base na estrutura interna do teste. Diante de um teste projetivo, e sabendo-se da complexidade da avaliação das propriedades psicométricas desses instrumentos, a identificação de resultados satisfatórios de evidência de validade de estrutura interna reforça a necessidade de seguir investindo neste instrumento e nos testes projetivos em geral, continuando acreditando no potencial dos dados que produzem.

Entende-se que a infância é uma fase crucial para o desenvolvimento dos sujeitos. Portanto, poder contar com instrumentos capazes de avaliar potencialidades, dificuldades e conflitos nessa fase da vida é, por si só, de grande relevância e importância. Acredita-se que, pela projeção, uma criança pode expressar o que sente e pensa. Assim, o teste projetivo pode ser considerado um facilitador/mediador que possibilita ao psicólogo compreender a dinâmica do funcionamento psíquico infantil, mas sempre sem perder de vista a idade, a fase desenvolvimental, a capacidade cognitiva e o meio familiar e social em que a criança está inserida (Werlang \& Cunha, 1993).

Para finalizar, parece relevante salientar que estudos deste porte possibilitam disponibilizar um teste psicológico de tipo projetivo que, futuramente, poderá ser utilizado pelos psicólogos brasileiros para a compreensão de alguns aspectos do mundo psíquico infantil com o objetivo maior de qualificação das avaliações e das intervenções que se venha a fazer com crianças dentro da prática em psicologia. Contudo, é importante mencionar algumas limitações do estudo aqui retratado. Embora não se tenha pretendido dar conta da totalidade dos estudos de validade, sabe-se da importância de se investir em novas pesquisas, na tentativa de buscar outras evidências de validade para o TCF.

\section{Referências}

American Educational Research Association, American Psychological Association \& National Council on Measurement in Education (1999). Standards for educational and psychological testing. American Educational Research Association: Washington DC.

Anderson \& Anderson (1966). Técnicas proyectivas del diagnostico psicológico. Madrid: Rialps.

Angelini, A. L., Alves, I. C. B., Custódio, E. M., Duarte, W. F., \& Duarte, J. L. M. (1999). Matrizes Progressivas Coloridas de Raven. Escala especial. São Paulo: Centro Editor de Testes e Pesquisas em Psicologia.

Anzieu, D. (1981). Os métodos projetivos. Rio de Janeiro: Campus.

Boorsboom, D., Mellenbergh, G. J. \& Van Heerden, J. (2004). The concept of validity. Psychological Review, 111(4), 1061-1071.

Conselho Federal de Psicologia - CFP. (2013). SATEPSI - Lista dos testes aprovados. Disponível em: http://www.pol.org.br. Acessado em: 24 jun. 2012.

Coulacoglou, C. (1995). Teste de los cuentos de hadas. Madrid: TEA Ediciones.

Coulacoglou, C. (2002a). Construct validation of the Fairy Tale-Test-Standardization data. International Journal of Testing. 2(3, 4) 217-242.

Coulacoglou, C. (2002b). Psychometrics \& Psychological Assessment. 2 nd. Ed. Athens: Paparazisis.

Coulacoglou, C. (2008). Exploring the child's personality: developmental, clinical and cross-cultural applications of the Fairy Tale Test. Springfielf: Thomas Books.

Coulacoglou, C. (2009). The Fairy Tale Test - Greek manual. Revised Edition (não publicado).

Coulacoglou, C., \& Souyouldjoglou, M. (2005). The Fairy Tale Projective Test as a means to examine psychoanalytic interpretations of fairy tales (Turkish article). Em Yanstima/Projection: Psychopathology and Projective Test, 2 (3-4), 173-189.

DeMers, S. T., Turner, S. M., Andberg, M., Foote, W., Hough, L., Ivnik, R., Meier, S., Moreland, K., \& Rey-Casserly, C. M. (2000). Report of task force on Test User Qualifications. (pp. 17-21). Washington: American Psychological Association. 
Fensterseifer, L., \& Werlang, B. S. G. (2008). Apontamentos sobre o status científico das técnicas projetivas. Em: A. E. Villemor-Amaral \& B. S. G. Werlang (Orgs.). Atualizações em Métodos Projetivos para Avaliação Psicológica (pp. 15-33). São Paulo: Casa do Psicólogo.

Formiga, N. S., \& Mello, I. (2000). Testes psicológicos e técnicas projetivas. Psicologia: Ciência e Profissão, 20(2): 12-19.

Montero, I., \& León O. G. (2005). Sistema de clasificación del Método en Los Informes de Investigación en Psicología. International Journal of Clinical and Health Psychology. 5(1), 115-127.

Pasquali, L. (2001). Técnicas de Exame Psicológico - TEP: manual. São Paulo, SP: Casa do Psicólogo; Conselho Federal de Psicologia.

Pasquali, L. (2003). Psicometria: teoria dos testes na psicologia e na educação. $2^{\mathrm{a}}$ Ed. Petrópolis: Vozes.

Primi, R., Muniz, M., \& Nunes. C. H. S. S. (2009). Definições contemporâneas de validade de testes psicológicos. Em: C. S. Hutz (Org.). Avanços e polêmicas em avaliação psicológica (pp. 243-265). São Paulo: Casa do Psicólogo.

Primi, R., Muniz, M.. \& Villemor-Amaral, A. E. (2009). Validade do Zulliger no Sistema Compreensivo. Em A. E. Villemor-Amaral \& R. Primi (Orgs). Teste de Zulliger no sistema compreensivo ZSC - forma individual (pp. 137-173). São Paulo: Casa do Psicólogo.

Spearman, C. (1927). The abilities of man: their nature and measurement. New York: Macmillan.

Urbina, S. (2007). Fundamentos da testagem psicológica. Porto Alegre: Artmed.

Villemor-Amaral, A. E., \& Primi, R. (2009). Teste de Zulliger no Sistema Compreensivo - ZSC: forma individual. São Paulo: Casa do Psicólogo.

Villemor-Amaral, A. E., \& Werlang, B. S. G. (Eds.). (2008). Atualizações em métodos projetivos para avaliação psicológica. São Paulo - SP: Casa do Psicólogo.

Werlang, B. S. G., \& Cunha, J. A. (1993). Avaliação da personalidade sob o enfoque projetivo. Em J. A. Cunha (Org.). Psicodiagnóstico-R (pp. 123-129). Porto Alegre: Artes Médicas.

Werlang, B. S. G., Villemor-Amaral, A. E., \& Nascimento, R. S. G. F. (2010). avaliação psicológica, testes e possibilidades de uso. Em Avaliação Psicológica: Diretrizes na Regulamentação da Profissão (pp. 87-100). Brasília: Conselho Federal de Psicologia.

Recebido em: 02/02/2012

Reformulado em: 02/04/2013 Segunda reformulação em: 11/09/2013 Aprovado em: 28/11/2013

Sobre os autores:

Blanca Susana Guevara Werlang é psicóloga, doutora em Ciências Médicas, área Saúde Mental pela Universidade Estadual de Campinas (UNICAMP), professora titular da Faculdade de Psicologia da PUCRS e membro da Comissão Consultiva em Avaliação Psicológica do Conselho Federal de Psicologia.

E-mail: bwerlang@pucrs.br

Carlos Henrique Sancineto da Silva Nunes é psicólogo, doutor em Psicologia pela Universidade Federal do Rio Grande do Sul (UFRGS), professor adjunto I da Universidade Federal de Santa Catarina (UFSC) e membro da Comissão Consultiva em Avaliação Psicológica do Conselho Federal de Psicologia.

E-mail: carloshnunes@mac.com

Vivian Roxo Borges é psicóloga, doutora em Psicologia pela Pontifícia Universidade Católica do Rio Grande do Sul (PUCRS), professora adjunta da Faculdade de Psicologia da PUCRS e conselheira titular do Conselho Regional de Psicologia do Rio Grande do Sul (CRPRS).

E-mail: vivian.borges@pucrs.br 